\title{
Data Collection using Rendezvous Points Tracking for Mobility enabled Wireless Sensor Networks
}

\author{
R. Sahila Devi, \\ B.E., M.Tech.,MISTE, \\ Assistant Professor/CSE, \\ PET Engineering College, \\ Tirunelveli, TamilNadu-627117
}

\author{
M. Anoop, \\ ME(CSE), \\ PET Engineering College, \\ Tirunelveli, \\ Tamil Nadu-627117
}

\author{
S.Sherin Liba, \\ ME(CSE) \\ PET Engineering College, \\ Tirunelveli, \\ Tamil Nadu-627117
}

\begin{abstract}
Energy saving can be achieved in mobility enabled wireless sensor networks that visit sensor nodes and collect data from them through short range communication. The problem that has been faced in WSNs is the increased latency in data collection due to the speed at which the data have been collected. So in order to collect the data efficiently a rendezvous point (RP) is used. Here data are collected by the base station while visiting the rendezvous points. The rendezvous points collect the data which are being buffered from various source nodes are being aggregated at a particular point known as RP. This work proposes an efficient rendezvous design algorithm with provable performance bounds with mobility and fixed tracking.
\end{abstract}

Keywords: Rendezvous point, mobility tracking, fixed tracking, mobility, Steiner minimum tree.

\section{INTRODUCTION}

The main problem faced in wireless sensor network is the network lifetime that must operate for an extended period of time on limited power supplies by means of batteries. To reduce the energy consumption of the WSN propose the rendezvous points which aggregate the data from various sources nodes and are being accumulated in one of the source node called as rendezvous point. The area in the network are grouped into various clusters and from each cluster a node which is close to the base station has been selected as the rendezvous point. The clusters are being created by means of partitioning.

The base station (BS) periodically visits the rendezvous points in each cluster and collects the data. Each cluster nodes in the network are connected like a binary tree; the edges of each node are connected by means of Steiner Minimum Tree (SMT). The tree is created by means of the shortest path between two nodes. The data are collected from various nodes in preorder traversal are aggregated in the RPs. since the data are collected in preorder the traffic will be reduced by avoiding the confusion in nodes to send the data to the RP. Before the base station starts tracking the data are cached in the RPS. The cached data are being taken by the BS and passes on to the next RP to take the next data that have been accumulated. Some of the advantages of this approach are,

- Use of rendezvous points will reduce the energy consumption and communication delay.

- Rendezvous points can collect large amount of data at a time from various source nodes in the cluster.
- $\quad$ Since the base station only visit the RPs will avoid the network disruption caused by mobility.

This section contributes the data collection by means of two approaches. The first describes about the fixed tracking (FT) in which the data are collected by means of fixed path. The second one describes about the mobility tracking (MT) in which the track is formed by means of the minimum distance. It has the following contributions they are, the rendezvous design problem for mobility enabled WSNs, which aims to find a set of RPs that can be visited by the BS within a required delay while the network cost in transmitting data from sources to RPs is reduced. Consider the Steiner Minimum Tree concept which aggregates the data efficiently at the RPs while shortening the data collection tour of BS. Simulation results show that both algorithms can achieve satisfactory performance under a range of settings. The theoretical performance bounds of the algorithms are also validated through simulation.

The main goal is to find the tour, which visits the set of nodes referred to as rendezvous points. The RPs collect the data originated at various nodes and send it to the base station by means of short range communication. A set of source node that generates data samples that must be delivers to the base station within the specified time interval. The use of RPs will minimize the network traffic, the length of the traversal path and the data will be delivered at particular deadline.

\section{LITERATURE SURVEY}

YaoyaoGu, DorukBozdag, et.al. Investigates a technique for partitioning-based algorithm is presented that schedules the movements of MEs in a sensor network such that there is no data loss due to buffer overflow [4]. Partitioning-Based Scheduling (PBS) algorithm performs well in terms of reducing the minimum required $\mathrm{ME}$ speed to prevent data loss, providing high predictability in inter-visit durations, and minimizing the data loss rate for the cases when the ME is constrained to move slower than the minimum required ME speed. The collected data at the sensors are usually transmitted to the sinks via power efficient multi-hop routing protocols. The traditional approach for data relaying in wireless sensor networks involves multi-hop communication from data sources to sinks.

Soo Kim, JeongGilKoet.al. Introduces the concept of Wireless Sensor Network (WSNs), energy efficiency and fault tolerance should be the two major issues in designing WSNs [7]. However, previous studies on positioning base stations in WSNs are focused on energy efficiency only. The two strategies to find the optimal position of BSs, 
- Minimizing the average transmission energy for energy efficiency;

- Minimizing additional energy consumption after a BS failure for fault tolerance

EylemEkici, YaoyaoGuet.al. Proposed Wireless sensor networks are proposed to deliver in situ observations at low cost over long periods of time [3].Maintaining connectivity and maximizing the network lifetime stands out as critical considerations. A large number of small and simple sensor devices communicate over short range wireless interfaces to deliver observations over multiple hops to central locations called sinks.

- The connectivity condition is generally met by deploying a sufficient number of sensors, or using specialized nodes with long-range communication capabilities to maintain a connected graph.

- The network lifetime can be increased through energy conserving methods such as using energyefficient protocols and algorithms, and battery replenishment techniques.

David Jea, ArunSomasundaraet.al describes a mobile element to collect and carry data mechanically from a sensor network has many advantages over static multihop routing the network scalability and traffic may make a single mobile element insufficient [8]. The nodes near the base station relay the data from nodes that are farther away. This leads to a non-uniform depletion of network resources and the nodes near the base station are the first to run out of batteries. The use of multiple mobile elements, load balancing algorithm which tries to balance the number of sensor nodes each mobile element services

Po-Liang Lin and Ren-Song Kop represents a typical WSNs use static sink to collect data from all sensor nodes via multihop forwarding, which can easily result in the hot spot problem since nodes close to the sink tend to consume more energy in relaying data from other nodes [9]. An MS can prevent the hot spot problem, but it takes time to move around. Reducing the number of points to traverse reduces the time needed to traverse them, thus, alleviating the buffer overflow problem. The traverse cost consists of two factors: buffer overflows time and Inter distance.

\section{PROPOSED SYSTEM}

This work proposes a rendezvous-based data collection approach. Specifically, a subset of static nodes in the network will serve as the rendezvous points which aggregate data originated from sources. The BS periodically visits each RP and picks up the cached data. In mobility tracking, the tracking is based on the shortest path from the base station to the rendezvous points and in fixed tracking the path is fixed and the base station track in the specified path chosen by the user. The partitioning based clustering method is used for grouping nodes and Steiner Minimum Tree algorithm is used for making the binary tree structure in each cluster. Some of the advantages are,

- A broad range of desirable trade-offs between energy consumption and communication delay can be achieved by jointly optimizing the choices of RPs, motion path of BS, and data transmission routes.

- The use of RPs enables the BS to collect a large volume of data at a time without traveling a long distance, which mitigates the negative impact of slow speed of BS on overall network throughput.

- Mobile nodes communicate with the rest of the network through RPs at scheduled times, which minimizes the disruption to the network topology caused by mobility.

- This method is robust in terms of high area coverage with a reasonable amount of active sensors and connectivity preservation despite message losses.

The approaches used for data collection in WSN consist of configuration model, clustering, SMT tree formation, RP calculation, fixed BS tracking, mobility BS tracking.

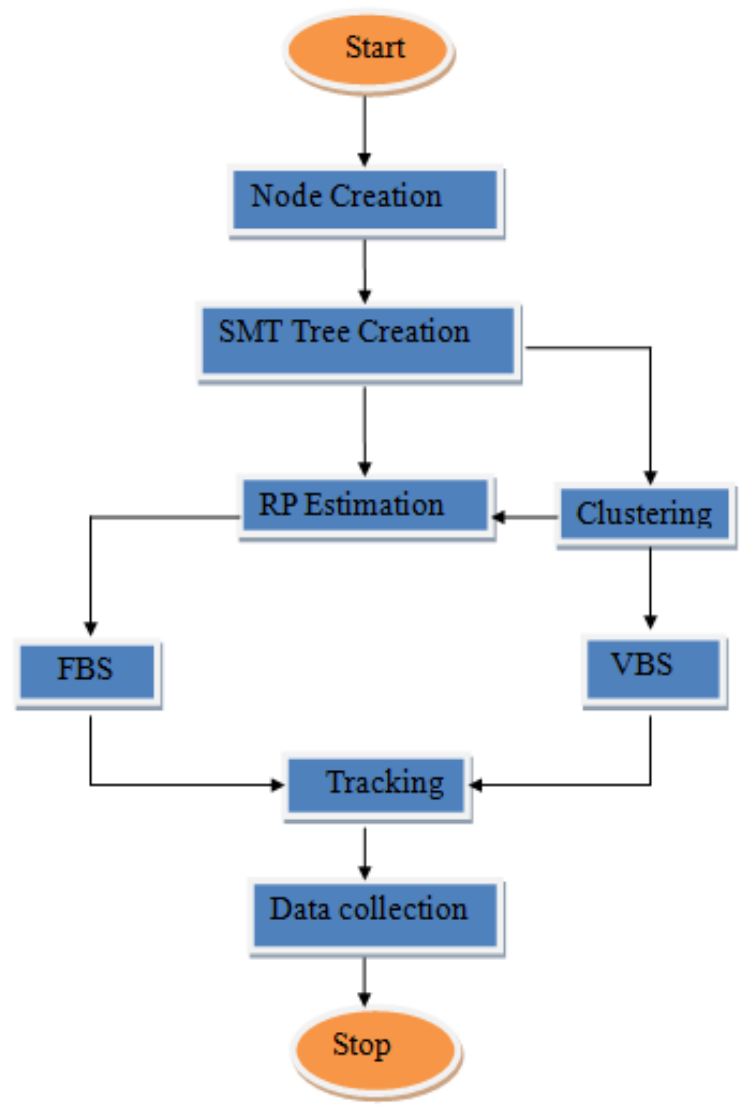

Figure 1. Flowchart for Rendezvous design.

\subsection{Configuration model}

This model designs the network model with number of nodes, node energy, and node position. The speed that data packets are relayed in a WSN is about several hundred meters per second, which is much higher than the speed that a mobile device moves. Therefore, the data collection deadline can be mapped to the maximum allowable length of the BS tour that visits all RPs. Each sensor node can be named by the user for the identification that which nodes send data to the RP. It assumes that data from different sources can be aggregated at a node before being relayed. Data aggregation has been widely adopted by data collection applications to reduce network traffic. The nodes are densely deployed in a region and all nodes use the same transmission power. The total energy consumed by transmitting a data packet along the path is proportional to the distance between the sender and the receiver 


\subsection{Clustering}

Clustering is the method adopted for seperating the nodes into various group that is a form of grouping nodes. This work includes partitioning based technique for clustering the source nodes. This may divide or group the nodes into various quadrants and this method is used to identify that how many nodes are being present in each quadrants. This can be used for identifying which node is closer to the base station for the purpose of finding RP and tracking for data collection. The sources nodes will be sending the data to the particular node that act as the RP in each quadrants.

\subsubsection{Algorithm}

- Analyse the coordinates of nodes on the network.

- Assume the plotting area with $\mathrm{W}$ and $\mathrm{H}$ as width and height.

- Calculate the centre of plotting area cx and cy where, $c x=W / 2$ and $c y=H / 2$.

- Analyse the nodes and find those with $x-$ coordinates $<c x \& y-$ coordinate $<c y$ and group them as top left grid.

- Analyse the nodes and find those with $x$ coordinates $\geq c x \& y-$ coordinate $\geq c y \quad$ and group them as bottom right grid.

- Analyse the nodes and find those with $x-$ coordinates $<c x \& y-$ coordinate $\geq c y \quad$ and group them as bottom left grid.

- Analyse the nodes and find those with $x-$ coordinates $\geq c x \& y-$ coordinate $<c y \quad$ and group them as top right right grid.

- If change in node position, repeat the steps

- $\quad$ Else end.

\subsection{SMT Tree Formation}

The tree is formed under the basic of kruskal's algorithm, in which the each nodes in the cluster are taken and the distance between each nodes will be calculated seperately. The shortest distance between the two nodes will be taken and the edges are drawn. The data will be passed to the RP in the preorder manner. There will be no confusion for the source nodes to send the data to the next node. The edges can be calculated absolutely. The starting node for the tree is selected randomly.

\subsubsection{Algorithm}

- Find an approximate Steiner minimum tree $\mathrm{T}$ that connects all point in S. Randomly choose a source B as the root of the tree.

- $\quad R=\varphi ; Y=0$

- $\quad$ while $(X=L-T S P(R)>\sigma)$

- $Y=Y+X / 2$;

- $\quad$ Traverse T from B in preorder until the total length of edges visited is Y. Denote subtree traversed as $T^{\prime}$

- $\quad \mathrm{R}=\left\{r_{i} \mid r_{i}\right.$ is the intersection between $\mathrm{t}$ and path $\mathrm{s} \rightarrow$ $\mathrm{B}$ on $\mathrm{T}\}$.

- $\quad$ end while

\subsection{Rendezvous point calculation}

This section describes about how to calculate the RP for the purpose of tracking. To place RPs on an approximate Steiner Minimum Tree of source nodes, which allows the data to be efficiently aggregated at RPs while shortening the data collection tour of base station. The RP point can be is assumed to be the shortest node in the cluster fron the base station. The RP in each cluster is described as the same. The source nodes will send the data to the RP and the RP can accumulate all the data sends by the source nodes. This uses the N-to- one aggregation model in which a node can aggregate multiple data packets it received into one packet before relaying it. The storage capacity of the nodes is large enough to buffer the total volume of data generated by the sources within delivery deadline. BS only visits the RP for data collection. The BS periodically visits each RP and picks up the cached data.

\subsection{Fixed BS Tracking}

This method analyzes the rendezvous design problem when the BS moves on a fixed track. Although a fixed track reduces the contacts between the BS and the fixed nodes in the network, it significantly simplifies the motion control of the BS and is hence adopted by several mobile sensor systems in practice. It assumes that the track consists of nonintersecting contiguous line segments, which is consistent to several practical mobile sensor systems. The objective is to find a continuous tour no longer than $\mathrm{L}$ along the track and a set of routing trees rooted on the tour that connect all sources, such that the total Euclidean length of the trees is minimized. The fixed track starts from the BS to all the RPs in various clusters. The user can define the path through which the tracking has been made.[BS- >RP1- >RP2 -> RP3 ->RP4]

\subsection{Mobility BS Tracking}

This method analyzes the rendezvous design problem when the BS can freely move in the network deployment region along any track. Our objective is to find a BS tour no longer than $\mathrm{L}$ and a set of routing trees that are rooted on the tour and connect all sources, such that the total Euclidean length of the trees is minimized. It develops the tracking by the shortest distance of the RP from the BS. When the first track has been drawn from the Bs to any one of the shortest RP, the RP will check for the next shortest RP in the network. After the track has been made the data are being collected by the Bs from the RP that had aggregated the data from various source nodes. [BS->RP2->RP3->RP4->RP1]

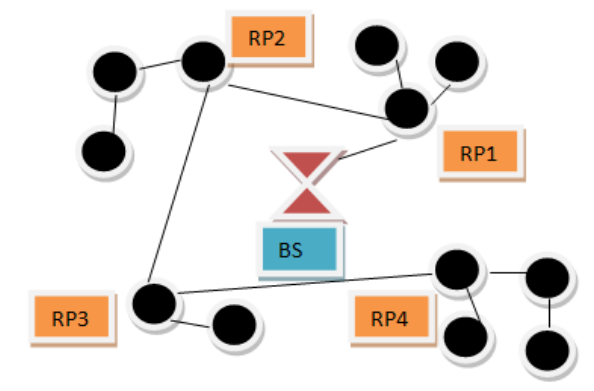

Figure 2. Overall design for Rendezvous design using clustering. 


\subsection{Data collection using single SMT:}

The data can be collected by the base station by means of fixed tracking in the network using single SMT creation. The SMT tree will be created by finding the shortest path between the nodes. The first node in the network will be taken randomly. The shortest edges will be joined together to form the SMT tree. Rendezvous points are created and we assume that the base station will traverse only to the RPs to collect the data. The tree has been traversed in the preorder manner that is root, left, right. The root node in the tree is considered as the RP points. The data are being collected by the BS by visiting the RP periodically. The RPs found in the network will not be constant. This may vary accordingly to the tree or the nodes that have been initialized.

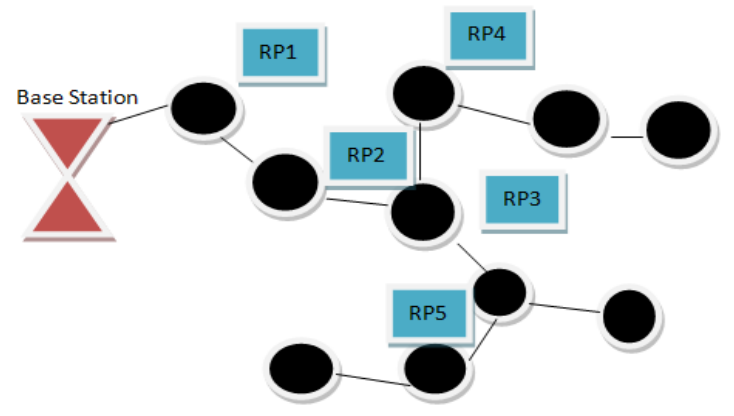

Figure 3. Data collection using single SMT tree.

\section{EXPERIMENTAL ANALYSIS}

This section evaluates the performance of rendezvous design algorithms. The simulations are based on a geometric network model in which the shortest point from the base station in the network region can be chosen as an RP. The performance metric is the total Euclidean length of routing trees that connect sources to the RPs. Such a geometric network model allows us to validate the design of MT and FT and the tightness of derived performance bounds.

Consider a graph $\mathrm{G}$, let $\mathrm{c}(\mathrm{G})$ represent the total edge length of G. The mobility tracking shows that all the found RPs can be visited by a Bs tour no longer than $\mathrm{L}$. $\mathrm{L}$ is the length of the BS tour.

$$
L=v_{m} D
$$

Where $\mathrm{D}$ is a data collection deadline, $v_{m}$ is the average moment speed of BS.

According to the definition of RPs, $R_{i}$ is a subset of vertices of $T_{i}$. If the tour found by the Travelling Salesman Problem (TSP) solver is longer than $2 \mathrm{c}\left(T_{i}\right)$, that is the total length of tour. Suppose $\operatorname{TSP}\left(R_{i}\right) \leq L$ holds in iterationi. For $i+1$ iteration, the formula is,

$$
c\left(T_{i+1}\right)-c\left(T_{i}\right)=\frac{L-T S P\left(R_{i}\right)}{2}
$$

A tour that visits all RPs in $R_{i+1}$ can be constructed by appending this preorder walk to the TSP tour in iteration $i$. The formula is,

$$
\begin{gathered}
\operatorname{TSP}\left(R_{i+1}\right) \leq \operatorname{TSP}\left(R_{i}\right)+2\left(c\left(T_{i+1}\right)-c\left(T_{i}\right)\right) \\
=T S P\left(R_{i}\right)+2 \cdot \frac{L-T S P\left(R_{i}\right)}{2}
\end{gathered}
$$

$=L$
SMTs denote the SMT connecting all sources $S=\left\{s_{i}\right\}$. Let $\beta$ be the ratio of $L$ to the total edge length of SMTs, that is

$$
\beta=\frac{L}{c(S M T s)} \quad \text { Where }, \beta \ll 1 .
$$

When this condition does not hold, the BS tour is close to or directly includes many source nodes.

Similarly, in fixed tracking a set of points are added on the track such that the segments between any two adjacent points has a length $\Delta L$. Denote $x_{k}$ as the subset of the added points that lie on tour $\rho_{k}$.for each tour $\rho_{k}$ find $S M T_{\rho_{k}}$ using the extended kruskal's algorithm. The final solution is the tour $\rho$ that minimizes the length of $S M T_{\rho}$ among all tours tested.

The source in $S$ connect to the track at $R=\left\{r_{1}, r_{2}, \ldots\right\}$ in $S M T_{\rho}$. Construct a tree (T) that connect sources in $S$ to points in $R$. Denote $T$ found as $T(S \cup R)$. Therefore

$$
c(T(S \cup R)) \leq \frac{2}{\sqrt{3}} \cdot c\left(S M T_{\rho}\right)
$$

The experimental analysis states that the total edge length of the routing tree for the given nodes is drawn with the help of SMT algorithm for mobility and fixed BS tracking is analyzed and shown below,

Table 1 shows the performance for different number of sources and the total edge length of routing tree for mobility tracking and fixed tracking it suffices only consider the sources closest point on the track as possible start points of BS tour, which significantly reduces the complexity of FT.

Table1.Performance evaluation of fixed and mobility tracking with BS

\begin{tabular}{|l|l|l|}
\hline $\begin{array}{c}\text { Number } \\
\text { of source } \\
\text { nodes }\end{array}$ & $\begin{array}{c}\text { Mobility } \\
\text { Tracking } \\
\text { "meter(m)" }\end{array}$ & $\begin{array}{c}\text { Fixed } \\
\text { Tracking } \\
\text { "meter(m)" }\end{array}$ \\
\hline 20 & 550 & 620 \\
\hline 30 & 630 & 710 \\
\hline 40 & 720 & 825 \\
\hline 50 & 800 & 960 \\
\hline 60 & 930 & 1035 \\
\hline 70 & 1010 & 1115 \\
\hline 80 & 1090 & 1265 \\
\hline 90 & 1200 & 1351 \\
\hline
\end{tabular}




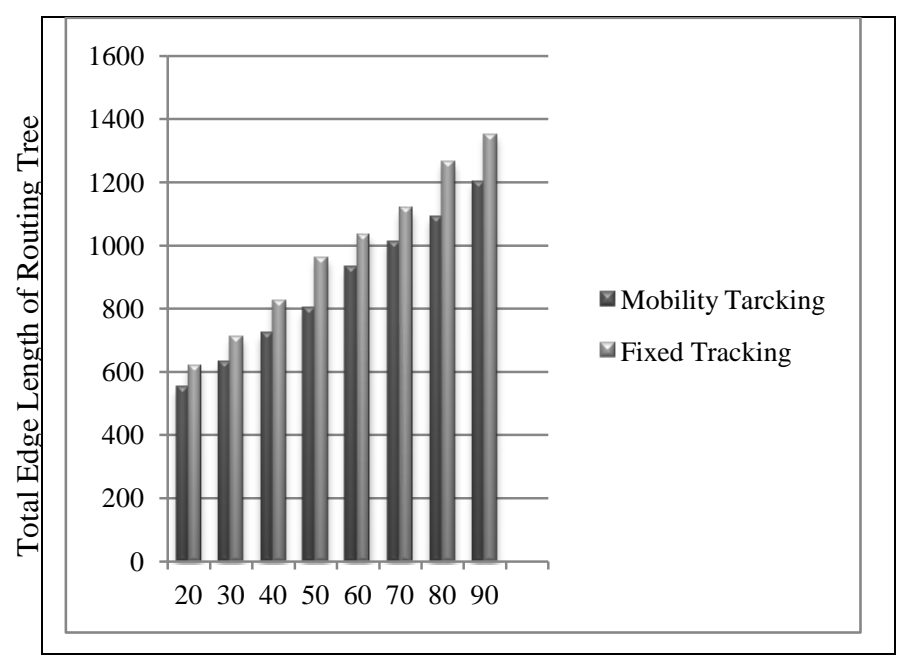

Number of Sources

Figure.4 Comparing thetotal edge length of routing treeand number of nodes for mobility and fixed tracking.

Table 2 shows the total edge length of routing tree and minimum length of BS tour for mobility tracking.It is a good approximation of SMT in the euclidean space.In the fixed BS tracking if the number of sources is more, then the satisfactory performance is obtained compared to mobility tracking.

Table2.Performance evaluation of minimum length of BS tour for fixed and mobility tracking.

\begin{tabular}{|l|l|l|}
\hline $\begin{array}{c}\text { Minimum } \\
\text { length of } \\
\text { BS tour }\end{array}$ & $\begin{array}{c}\text { Mobility } \\
\text { Tracking } \\
\text { "meter(m)" }\end{array}$ & $\begin{array}{c}\text { Fixed } \\
\text { Tracking } \\
\text { "meter(m)" }\end{array}$ \\
\hline 200 & 795 & 859 \\
\hline 300 & 790 & 858 \\
\hline 400 & 695 & 842 \\
\hline 500 & 585 & 830 \\
\hline 600 & 512 & 822 \\
\hline 700 & 450 & 811 \\
\hline 800 & 411 & 804 \\
\hline 900 & 358 & 790 \\
\hline 1000 & 292 & 785 \\
\hline
\end{tabular}

Figure.5 Comparison of the minimum length BS tour between RPS for mobility and fixed tracking.

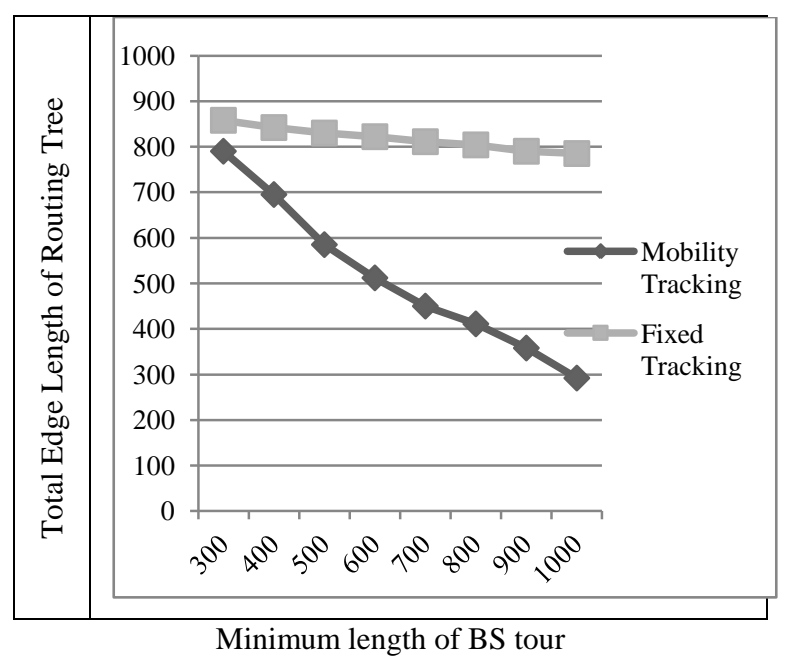

\section{CONCLUSION AND FUTURE WORK}

This work provides the rendezvous based data collection in WSN with mobile BS. The efficient redezvous algorithms with constant approximation ratios for two different cases are done (i.e) mobile BSs can move freely in the deployment region or must move along fixed track to collect the datas from theRPs efficiently. Simulation results shows that our algorithm can achieve satisfactory performance under a range of settings.

The future works include usage of multiple base stations (BS) in fixed and mobility tracking for data collection effectively with short time span. The future work can have many of the different traversal methods to collect the available data. The future work allows the nodes to transfer the data effectively by avoiding the traffic to reach the rendezvous points without data loss. This also includes the custom data transfer by the nodes to reach the destination in periodic intervals. This can include the Time To Live (TTL) value for each nodes before sending the data to the rendezvous points. Thereby the data available at the Rendezvous Point can be valid and real.

\section{REFERENCES}

[1] David Jea, Arun Somasundara, Mani Srivastava, 2009Multiple Controlled Mobile Elements (Data Mules)for Data Collection in Sensor Networks, UCLAfdcjea,arun,mbsg@ee.ucla.edu.

[2] Eylem Ekici, Yaoyao Gu, and Doruk Bozdag,july2006 Mobility Based Communication in Wireless Sensor Networks, Ohio State University.

[3] D. Li, X. Jia, and H. Liu, Apr.-June 2004. "Energy Efficient Broadcast Routing inAd Hoc Wireless Networks," IEEE Trans. Mobile Computing, vol. 3, no. 2,pp. 144-151.

[4] H. Liu, X. Jia, P. Wan, C. Yi, S. Makki, and N. Pissinou, Apr. 2007,"Maximizing Lifetime of Sensor Surveillance Systems," IEEE/ ACM Trans. Networking, vol. 15, no 2,pp. 334345 
[5] J. Luo and J.-P.Hubaux,2005, "Joint Mobility and Routing for Lifetime Elongation in Wireless Sensor Networks," Proc. IEEE INFOCOM.

[6] Po Liang Lin and Ren-Song Ko, August 2011, An Efficient Data Gathering Scheme for Heterogeneous Sensor Networksvia Mobile Sinks.

[7] A.A. Somasundara, A. Ramamoorthy, and M.B.Srivastava, Apr. 2007,MobileElement Scheduling with Dynamic Deadlines, IEEETrans. Mobile Computing, vol.6, no. 4,pp. 395-410.
[8] Soo Kim, Jeong Gil Ko, Jongwon Yoon, and HeejoLee ,2008, Energy-Efficient and Fault-Tolerant Positioning ofMultiple Base Stations Korea university, \{sooo,jgko,yoonj,heejo\}@ korea.ac.kr.

[9] YaoyaoGu, DorukBozdag , Robert W. Brewer, March 2006,EylemEkici, Data harvesting with mobile elements in wireless sensor networks. 